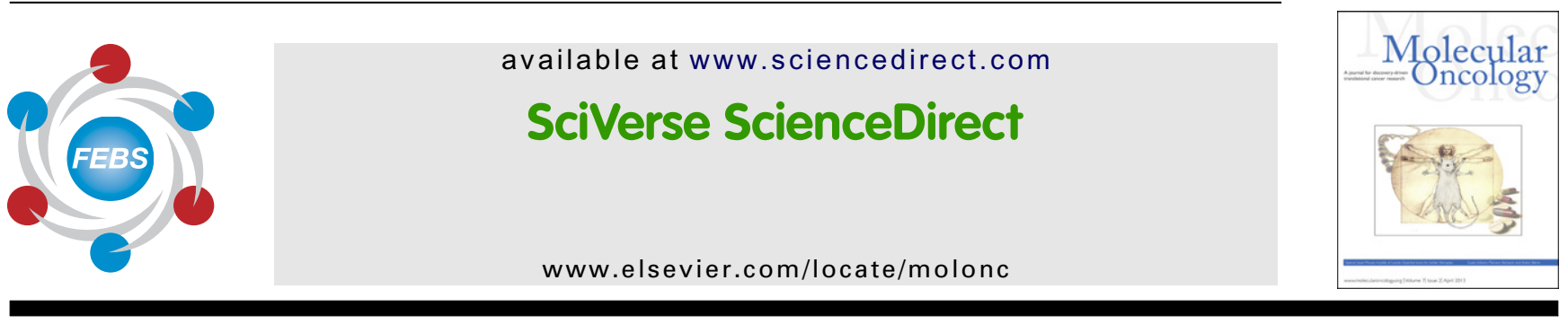

Editorial

\title{
Mouse models of cancer
}

\section{Foreword}

In this thematic issue of Molecular Oncology you will find a compendium of reviews on mouse models of cancer written by experts in the field. The various chapters deal with mouse models engineered to gain deeper insight into what drives tumor initiation and tumor progression, an aspect that is difficult to study in human tumors. The reviews primarily focus on modeling studies of frequently occurring human tumors. Each review highlights what they have taught us and also how this knowledge has already contributed or can contribute to the treatment of cancer patients. In all cases, the mouse models described in this thematic issue have been carefully crafted to mimic as much as possible the natural history of the human disease. In the past, mouse models of cancer were primarily designed to develop tumors in the most efficient way paying limited attention to the overall properties of the tumor or to the nature of their driver mutations. The advent of sophisticated gene-targeting technologies are now making it possible to design mouse models of cancer that faithfully recapitulate not only the anatomo-pathological changes characteristic of key tumor types but also those mutational events known to be responsible for the onset and subsequent development of human cancer. Thus, these models offer experimental systems that within the obvious limitations do reproduce human cancer with a level of similarities that was not even conceived to be possible just a decade ago. We trust that the reviews contained in this issue monographic issue will provide the reader with a comprehensive overview of the field illustrating the relevance of mouse models to improve our understanding of how cancer develops using in vivo models amenable to sophisticated experimentation.

These reviews will illustrate how these models not only provide a window to study the tumorigenic process within the tumors cells, but they also offer suitable models to understand the multiple events that accompany tumor development, events now generically known as tumor microenvironment. Expression of oncogenic mutations in selected cell populations allow tumors to proceed in a slow, step-wise mode that allow the host organism to respond by developing not only inflammatory and angiogenic responses, but also the generation of stroma and other tumor associated cells that closely reproduce those events commonly observed in human cancers. These properties set apart the genetically engineered mouse models (GEMMs) from all the other experimental systems designed to study human cancer in a laboratory setting.

In addition to faithfully reproduce human cancer in an in vivo experimental setting, GEMMs offer a wide range of possibility to test novel therapeutics in pre-clinical, co-clinical and post-clinical trials should serve as an invaluable tool to complement those clinical trials that eventually will serve to bring new and better medicines to treat cancer patients. Without doubt, our knowledge of cancer has grown enormously in the last decade. However, while digging deeper to understand its causes, we increasingly realize that cancer development is a far more complicated process than we had anticipated. Based on the knowledge of frequently occurring genetic defects that drive tumor development, we have developed new targeted drugs that do what we expected: impairing the activity of oncogenic proteins on which the tumor depends for its growth and maintenance. Some of these drugs showed spectacular effects when administered to cancer patients, including some complete regressions. However, the effects are generally very temporary and the tumors recur, often becoming refractory to the drug that was so successful initially. Massive parallel sequence analyses of human tumors have taught us a reason behind this disturbing observation, which is reminiscent of the resistance that was also often seen in patients that responded to chemotherapy. Tumors show extensive heterogeneity displaying, almost invariably, small populations of tumor cells that are refractory to the drug due to secondary mutations. Often, one can find in the tumor multiple subclones that have undergone convergent evolution carrying different mutations in the same oncogenic protein or in proteins that deregulate the same signaling pathway. In addition, epigenetic heterogeneity inherent to any cell population can make tumors refractory to many different drugs, providing a mechanism by which tumor cells might survive treatment.

Although this heterogeneity is disturbing from a therapeutic defined-target perspective, the observed convergence does 
offer treatment perspectives as it indicates that a particular tumor is apparently heavily addicted to the deregulation of distinct signaling pathways and that this might be hard wired. However, in order to effectively exploit this, we need to meticulously identify how the underlying signaling networks function and find out how to disrupt them, may be in combination with epigenetic drugs to prevent escapes driven by epigenetic mechanisms. In addition to the mutations clustering in specific signaling networks, we also observe many additional mutations in human tumors that could serve as important drivers of tumorigenesis. We need systems to test how important these mutations are for tumor maintenance. Given this extensive heterogeneity, we need to identify critical driver pathways and learn at what node we can interrupt them using specific drugs. Ultimately, it is almost certain that we will need to administer several drugs inhibiting multiple components of a distinct signaling network and target more than one network. Assuming that we have identified such pathways the immediate question that then comes up is which drugs to use to effectively control those pathways, what are the associated toxicities, and how do we have to schedule those if multiple networks need to be controlled while keeping toxicities within acceptable limits. We impossible can explore this thoroughly in clinical trials.

In this challenging scenario GEMMs should play a key role in advancing novel therapeutic strategies. Mouse models can teach us how combination treatments can be best given and provide us with mechanistic insights behind failure and success. First of all, targets can be validated using genetic approaches that do not require drugs, hence providing preclinical information that will help pharma to exclusively focus on those pathways that are really essential for tumor development in an in vivo setting that closely reproduce human cancers. Once drug candidates are available, they can be tested in tumors of varying aggressive properties by using GEMMs with varying combinations of driver mutations. Compounds that fail to produce tumor regression when tested against very advanced tumors may have therapeutic benefit when tested against less aggressive tumors induced by single or limited driver mutations. These compounds, otherwise discarded early in the process of drug discovery, might be extremely useful in combination with other drug candidates affecting complementary and/or independent oncogenic pathways. Moreover, as illustrated in some of these reviews, genetic approaches will reveal potential toxic effects that might serve investigators to separate mechanism-based toxicities from those derived from secondary effects due to the limited selectivity exhibited by many drug candidates.

Systematic approaches in pre-clinical trials using GEMMs carrying selected driver mutations are more likely to provide insights into combination therapies than current xenograft models in which tumors of human origin grow rapidly (weeks instead of years), lack of a natural microenvironment and may not represent the original tumors due to the accumulation of spurious mutations during experimental passages. These properties might certainly explain the limited predictability of the results obtained when transferred to the clinic. This limited predictability has unfairly given a bad name to all mouse tumor models and have led several pharma companies to accelerate the use of drug candidates in the clinic with, for the most part, disastrous results. The recent development of patient-derived xenograft (PDX) models has circumvented the problem of long term passage of human tumor tissue. However, these tumor models are not amenable to systematic analysis and hence they are unlikely to unveil provide therapeutic strategies that could be applicable to large cohort of patients.

In addition to their obvious utility in pre-clinical assays, GEMMs can also be used in co-clinical trials in which trials in patients are flanked by parallel trials in autochthonous mouse models in which the same therapeutic regimen is used. This can provide important mechanistic insight in treatment success or failure. Especially for combination therapies we need to learn in which way drugs can best be given such as dose, frequency, order and timing. In addition, GEMMs can be used to address questions difficult to resolve using human material such as biomarker development and validation as well as to have access to tumor biopsies in which to ascertain pathways activity and specificity of the tested drug(s). GEMMs can also advance crucial information regarding resistance mechanisms thus providing critical information to medical oncologists regarding potential treatments to overcome the growth of resistant tumors in patients before they acquire irreversible malignant properties. Mouse models might also teach whether a particular treatment can eliminate the cancer-initiating cell compartment, an aspect this is almost impossible to monitor directly in patients. The autochthonous mouse models currently available are also an exquisite source of defined tumor cell lines that can be used in drug response and resistance screens using drug and shRNA library screening pipelines. The latter can be used both to identify resistance mechanisms as well as targets to overcome resistance. Drop-out shRNA screens conducted both in vitro in cell lines and in vivo in tumor cell graft models can yield promising synthetic lethal targets for intervention.

Finally, GEMMs can also offer valuable information in postclinical trials to understand why a particular clinical trial might have failed. Considering the high attrition rate of clinical trials in oncology, understanding the reasons why a trial was not successful may provide very valuable information to prevent making similar mistakes in the future or to unveiled potential resistance mechanisms that might have been previously unsuspected due to the limited possibilities to work with human samples, especially after a clinical trial has been closed.

In summary, we hope that the information contained in this monographic issue of Molecular oncology should make it evident to academic scientists interested in understanding the molecular intricacies of tumor initiation and progression, to pharma scientists and executives actively engaged in identifying effective therapeutic strategies and to policy makers, that making mouse models that faithfully recapitulate human tumors is an indispensible tool to make substantial progress in identifying better medicines and better treatments for cancer patients. This is an area where we need the support of science policy makers responsible for assuring that we make the most effective use of public funds to fight cancer. It is our joint responsibility to guarantee adequate continuity of high 
quality infrastructures and expertise so that these tumor models can be effectively used to design new and better treatments that will ultimately benefit cancer patients.

Anton Berns* Division of Molecular Genetics, The Netherlands Cancer Institute, Plesmanlaan 121, 1066 CX Amsterdam, The Netherlands *Corresponding author. Tel.: +31 20 5121991; fax: +31 20

E-mail address: a.berns@nki.nl
Mariano Barbacid

Molecular Oncology Program, Centro Nacional de Investigaciones Oncologicas (CNIO), Madrid E-28029, Spain E-mail address: barbacid@cnio.es

1574-7891/\$ - see front matter (C) 2013 Federation of European Biochemical Societies. Published by Elsevier B.V. All rights reserved. http://dx.doi.org/10.1016/j.molonc.2013.02.014 